\title{
Tuning the Properties of Luminescent Nitrogen-Doped Carbon Dots by Reaction
}

\section{Precursors}

Hui Peng, ${ }^{a, b, * * \dot{*}}$ Ying Li, ${ }^{a, \dot{*}}$ Chunli Jiang, ${ }^{a}$ Chunhua Luo, ${ }^{a}$ Ruijuan Qi ${ }^{a}$ Rong Huang, ${ }^{a}$ ChunGang Duan ${ }^{a}$ and Jadranka Travas-Sejdic ${ }^{b, c}$

${ }^{a}$ Key Laboratory of Polar Materials and Devices, Ministry of Education, East China Normal University, Shanghai, P. R. China

${ }^{\mathrm{b}}$ Polymer Electronic Research Centre, The University of Auckland, Private Bag 92019, Auckland, New Zealand.

${ }^{\mathrm{c}}$ MacDiarmid Institute for Advanced Materials and Nanotechnology, New Zealand KEYWORDS. Nitrogen-doped carbon dots, pH-sensitivity, Electrochemical activity, Solar cell.

ABSTRACT: Four types of luminescent nitrogen-doped carbon dots (C-dots) are prepared by using glucose $(G)$ or citric acid $(C)$ as carbon source, and dopamine $(D)$ or 4,7,10-trioxa-1,13tridecanediamine $(T)$ as nitrogen source, named $G D$-C-dots, $G T$-C-dots, $C D$-C-dots and $C T$-Cdots. The investigation on the optical, $\mathrm{pH}$-sensitivity and photovoltaic properties of these C-dots illustrates that nitrogen source plays a key role in the properties of obtained C-dots. $G D$-C-dots and $C D$-C-dots show interesting electrochemical activities, which can provide fluorescent and electrochemical dual signals for practical applications. We demonstrate that not only the optical properties but also other physicochemical properties can be tuned by appropriate choice of the reaction precursors.

*Corresponding author. Email: hpeng@ee.ecnu.edu.cn (Hui Peng)

† H. Peng and Y. Li contributed equally. 


\section{Introduction}

As a member of the carbon -based nanomaterials family, luminescent carbon dots (C-dots) have drawn more and more attention in recent years due to their easy preparation[1-4], low biological toxicity [5-7] and comparable photoluminescence properties with conventional semiconductor quantum dots (QDs) [8, 9]. C-dots generally comprise discrete, quasispherical nanoparticles with sizes smaller than $10 \mathrm{~nm}$ and typically display size and excitation wavelength dependent photoluminescence (PL) behavior [10]. Until now, great successes have been achieved on the application of $\mathrm{C}$-dots as luminescent probes for biosensors $[11,12]$, bioimaging [13-15], drug delivery $[6,16,17]$ and optoelectronic devices $[18,19]$.

Various methods have been developed for the preparation of luminescent C-dots in recent years. Examples include C-dots derived from graphite powder [20], candle soot [21], and carbohydrate [22], thermal treatment of organic precursors, [2, 4, 9, 23], ultrasonic treatment of active carbon,[3], UV irradiation of organic precursors at room temperature [24] and electrochemical oxidation of carbon fiber [25] and graphite [26]. Among these methods, thermal treatments of organic precursors are appealing due to their simplicity, versatility and low cost of raw materials. As carbon source, different organic precursors have been reported to produce Cdots, such as glucose [4, 13, 27], citric acid [9], acetic acid [14], amino acid [28], even protein [29], egg [30] and grass [31, 32]. However, the C-dots prepared from different precursors show diverse properties, even those prepared from the same precursors but via different reaction pathways [4, 13]. For example, Wei et al. [4] used glucose and different amino acids via Maillard reaction to produce $\mathrm{C}$-dots with multi-emission colors under the same excitation wavelength. Another case is that of carbohydrates treated in octadecylamine and octadecene solution at 80 ${ }^{\circ} \mathrm{C}$, resulting in hydrophobic C-dots with blue or green emission, while hydrophilic C-dots with 
blue, green, yellow or red emission were produced by treating the same materials with concentrated sulphuric acid or concentrated phosphoric acid [13].

Although great successes have been achieved, there are still some challenges in the thermalbased treatment methods for the preparation of C-dots. Due to the complexity of carbonization process of organic precursors which results in the different composition of C-dots, a complete understanding of the photoluminescence (PL) origins in C-dots is still in question, which significantly hinders the development of C-dots with desired PL characteristics. Though few mechanisms based on particle-size distribution, surface traps and quantum effect [33-35], charge transfer [36], formation of aromatic molecules [37] and radiation recombination of excitations $[38,39]$, have been proposed, there is no universally accepted interpretation in the literature so far. Another challenge is how to choose precursors to prepare C-dots with desired properties, not only PL properties but also other physicochemical properties such as $\mathrm{pH}$ sensitivity and photovoltaic properties.

Glucose and citric acid are widely used as a carbon source in the preparation of C-dots $[4,9$, $13,35,40-42]$. In this work, we elucidate the role of these compounds as the carbon source relative to the role of compounds that may act as a nitrogen source, an important question not well understood so far. We prepared nitrogen doped C-dots by the thermal-treatment of glucose and citric acid in the presence of 4,7,10-trioxa-1,13-tridecanediamine (TTDDA) or dopamine which act as nitrogen source. For convenience, the C-dots prepared from glucose and citric acid in the presence of dopamine or TTDDA are nominated as $G D$-C-dots, $G T$-C-dots, $C D$-C-dots and CT-C-dots, respectively. As will be seen, glucose and citric acid show little effect on the properties of prepared C-dots, while TTDDA and dopamine which are designed as a nitrogen source play a key role in the properties of the prepared C-dots. They affect not only the optical 
properties, but also other properties, such as $\mathrm{pH}$-sensitive. Interesting, the C-dots prepared in the presence of dopamine show considerable electrochemical activity, which provides a strategy to one-step synthesis of multiple functionalized C-dots.

\section{Experimental}

\subsection{Materials}

Glucose, citric acid and dopamine were purchased from Aladdin Chemistry Co., Ltd (Shanghai, China). 4,7,10-Trioxa-1,13-tridecanediamine ( 97\% , TTDDA) was obtained from SigmaAldrich Co., Ltd. Spectra/Por molecularporous membrane tubing (MWCO 1000) was purchased from 3Bio Co., Ltd (Shanghai, China). All chemicals were analytical grade or better and used without further purification.

\subsection{Synthesis of $C$-dots}

For the preparation of $G T$-C-dots, $11 \mathrm{~g}$ of glucose was added into a round-bottom flask containing $27 \mathrm{~mL}$ of TTDDA. The mixture was heated to $250^{\circ} \mathrm{C}$ by using a sand bath and kept for one hour under nitrogen atmosphere. After reaction, the resulting mixture was dispersed in 10 $\mathrm{mL}$ of deionized water and sonicated for $30 \mathrm{~min}$. After centrifugation at $8000 \mathrm{rpm}$ for $10 \mathrm{~min}$, the supernatant was transferred to a dialysis membrane tube and dialyzed in flowing water for 24 hours to remove unreacted species. The preparation of $C T$-C-dots is similar to GT-C-dots, except that citric acid (12.9g) was used instead of glucose.

For the preparation of $G D$-C-dots, $0.45 \mathrm{~g}$ of glucose and $0.48 \mathrm{~g}$ dopamine were first dissolved in $20 \mathrm{~mL}$ deionized water, and then the solution was heated to $250^{\circ} \mathrm{C}$ by using a sand bath and kept for one hour under a nitrogen atmosphere. After reaction, the collection and purification of 
$G D$-C-dots were the same as for $G T$-C-dots. The preparation of $C D$-C-dots was similar to $G D$-Cdots, except that citric acid $(0.5 \mathrm{~g})$ was used instead of glucose.

\subsection{Characterization}

UV-visible absorption spectra and PL spectra were measured by using a UV-visible spectrophotometer (TU-1901, Puxi Beijing) and a Perkin Elmer LS55 fluorescence spectrometer, respectively. The X-ray diffraction patterns were recorded by a Bruker D8 X-ray diffractometer using $\mathrm{Cu} \mathrm{K} \alpha$ radiation source $(\lambda=1.541 \AA)$. The XRD scans were collected from $10^{\circ}$ to $70^{\circ}$

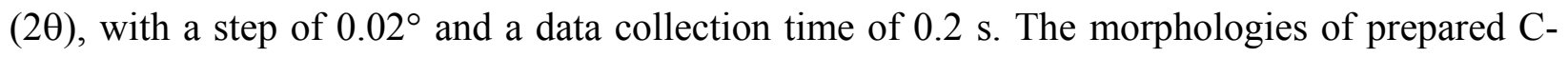
dots were characterized by a transmission electron microscopy (TEM, JEM 2100F, Japan). Atomic force microscopy (AFM) measurements were performed using a Nanoscope V multimode atomic force microscope (Veeco Instruments, USA) under ambient conditions, and samples were prepared by dropping the solution on the substrate of mica. The elemental compositions $(\mathrm{C}, \mathrm{H}$ and $\mathrm{N})$ of prepared $\mathrm{C}$-dot were analyzed by using a Vario EL III Element Analyzer (Germany). The contain of oxygen was obtained by calculation. The cyclic voltammograms were measured by using an electrochemical workstation (Autolab NOVA, Metrohm) with a conventional three electrode system including a glassy carbon electrode, an $\mathrm{Ag} / \mathrm{AgCl}(3 \mathrm{M} \mathrm{NaCl})$ reference electrode and a platinum wire counter electrode.

\section{Results and discussion}

The morphologies of all C-dots were first characterized by transmission electron microscopy and atomic force microscopy. The typical TEM images of $G T$-C-dots, $C T$-C-dots, $G D$-C-dots and $C D$-C-dots are shown in Figure 1. All of the four C-dots have a quasi-spherical shape with diameters in the range of $2 \mathrm{~nm}$ to $7 \mathrm{~nm}$. The AFM images (Figure 2) reveal that the heights of C- 
dots are in the similar range, further confirming the quasi-spherical shape of the prepared C-dots. High resolution TEM images illustrate crystallinity of the prepared C-dots, as shown in the inset of Figure 1. The lattice parameters of $G T$-C-dots, $C T$-C-dots, $G D$-C-dots and $C D$-C-dots are $c a$. $0.24,0.25,0.21$ and $0.25 \mathrm{~nm}$, respectively, which are different to the intra- plane lattice parameter $(0.214 \mathrm{~nm})$ and inter-plane lattice parameter $(0.340-0.403 \mathrm{~nm})$ of the reported C-dots derived from carbon sources without nitrogen doping [43-45]. The mismatch of lattice parameters could be due to the presence of nitrogen and oxygen, which have also been observed in other nitrogen- or oxygen- rich C-dots $[4,46]$. The XRD patterns are shown in Figure S1. For all of four samples, two broad peaks were found located at around $26^{\circ}(002)$ and $45^{\circ}(100)$, respectively. The size distributions of these four types of C-dots (Figure 1E-H) indicate that both

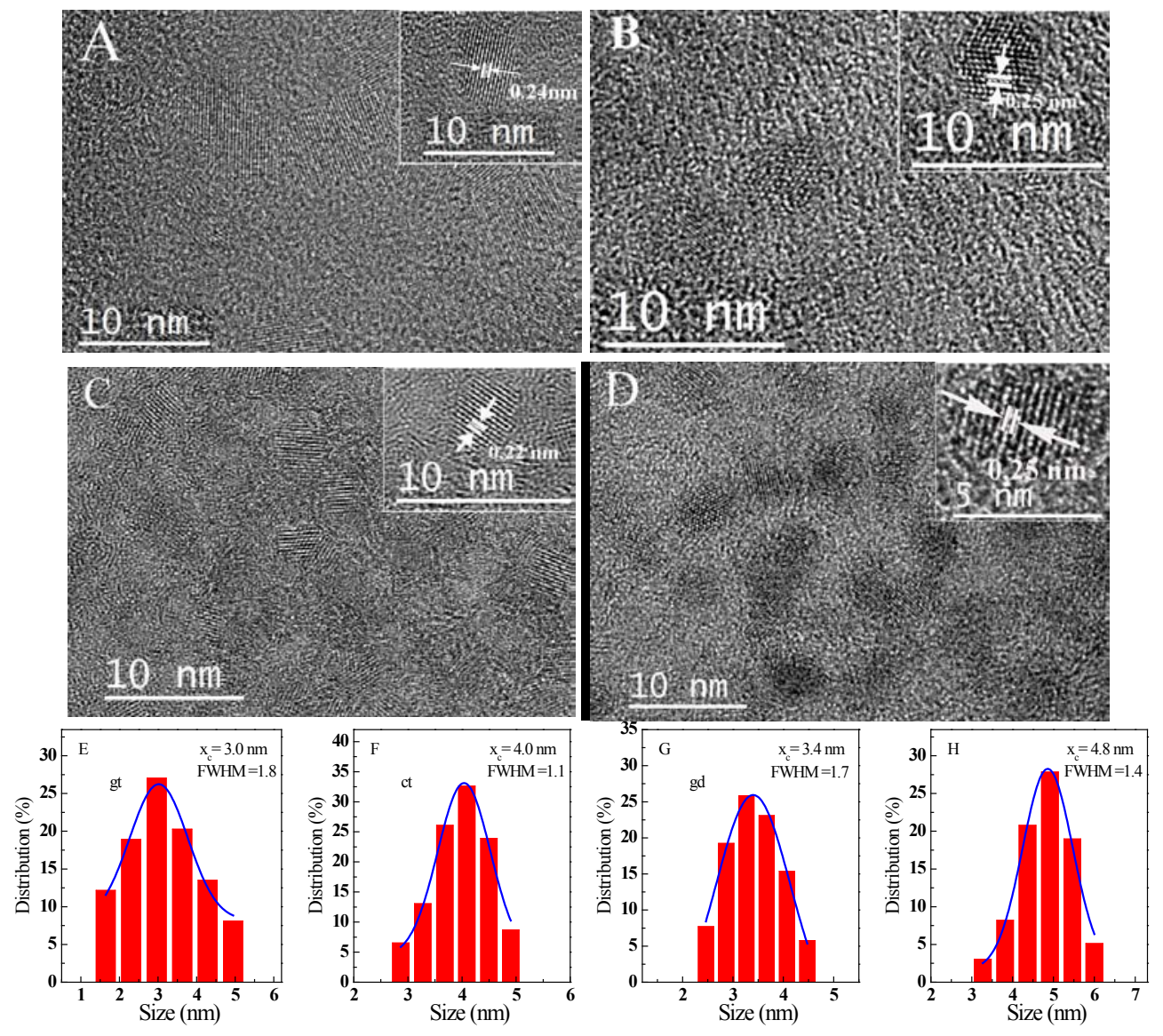

Figure 1 TEM images of (A) $G T$-C-dots (B) $C T$-C-dots (C) $G D$-C-dots and (D) $C D$-C-dots. 
carbon sources (glucose and citric acid) and nitrogen sources (TTDDA and dopamine) contribute to the size of C-dots. The average sizes of C-dots derived from citric acid are obviously larger than those derived from glucose. This could be attributed to the fact that citric acid possesses carboxyl groups that can react with basic TTDDA or dopamine to form stable intermediates. Contrary to that, glucose that has only hydroxyls groups can only interact with TTDDA and dopamine through Van der Waals' forces such as hydrogen bonds. The weak interaction between glucose and TTDDA or dopamine causes the intermediates to easily slip into small fragments during the heat-treatment process, resulting in smaller C-dots. By using the same carbon source, the average size of $\mathrm{C}$-dots derived from dopamine is somewhat larger than that derived from TTDDA, possibly due to dopamine containing phenyl ring.
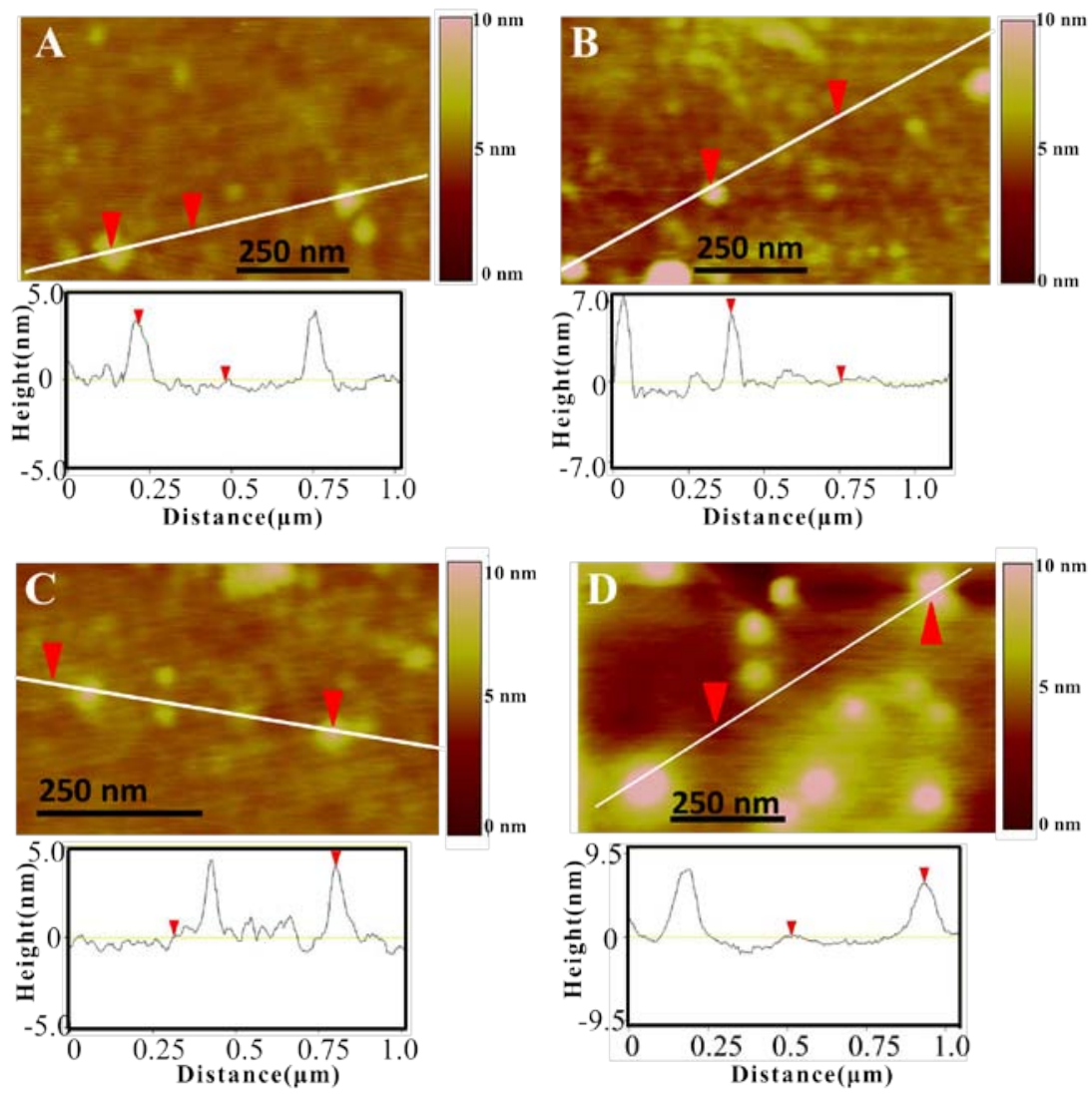

Figure 2 AFM images of (A) GT-C-dots, (B) $C T$-C-dots, (C) $G D$-C-dots and (D) $C D$-C-dots 

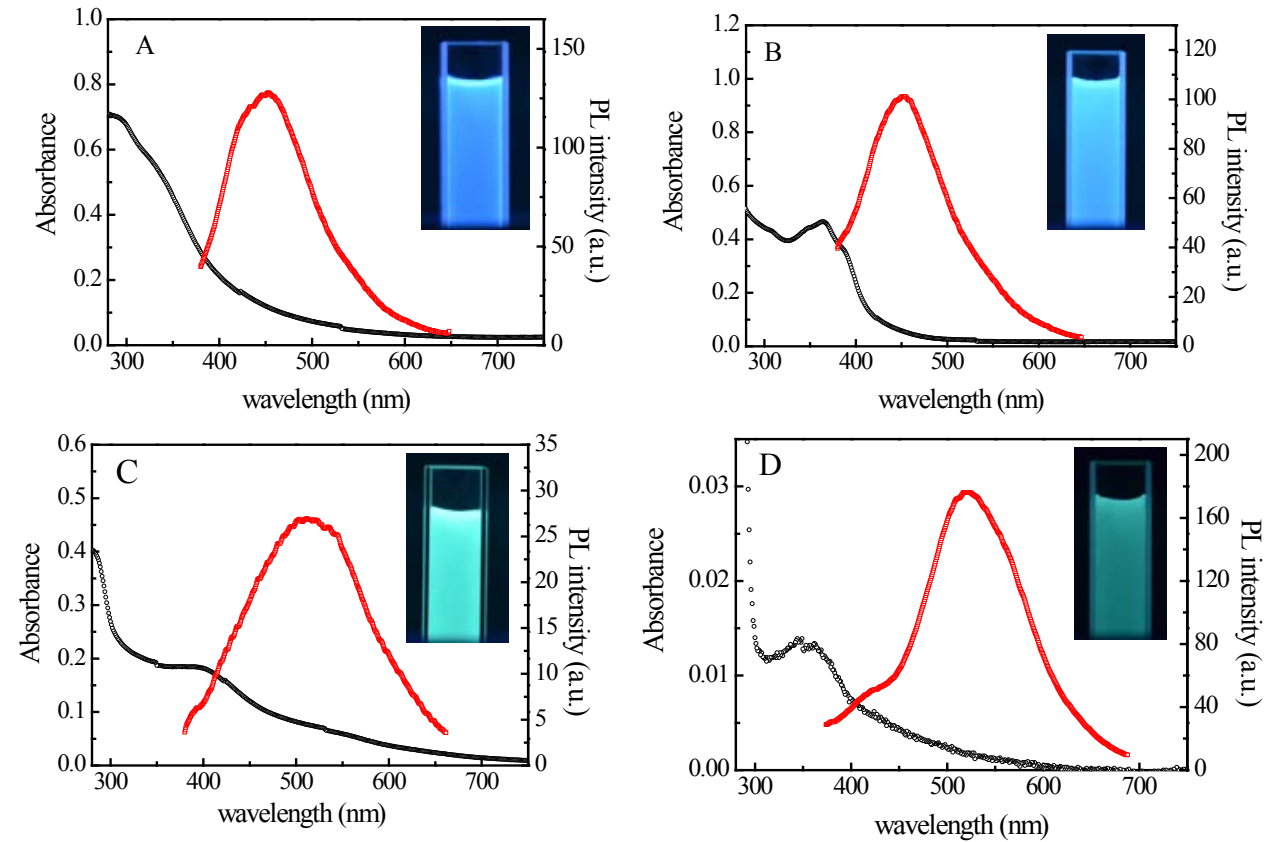

Figure 3 Absorption spectra and emission spectra of (A) GT-C-dots, (B) CT-C-dots, (C) GD-Cdots and (D) $C D$-C-dots. The emission spectra were recorded at an excitation wavelength of 340 $\mathrm{nm}$. Insets: the optical images of prepared C-dots under UV lamp (324 nm).

The optical properties of these four types of C-dots were investigated to identify the contributions of the reaction precursors. The UV-visible absorption and photoluminescence emission spectra are presented in Figure 3. The C-dots show a strong absorption feature around $340 \mathrm{~nm}$ in the UV-vis absorption spectrum, except $G T$-C-dots. The emission peak wavelengths of $G T$-C-dots, $C T$-C-dots, $G D$-C-dots and $C D$-C-dots under an excitation wavelength of $340 \mathrm{~nm}$ are $454 \mathrm{~nm}, 453 \mathrm{~nm}, 515 \mathrm{~nm}$ and $509 \mathrm{~nm}$, respectively. It is clear that C-dots prepared in the presence of TTDDA emit blue light and C-dots prepared in the presence of dopamine emit green light. The fluorescence quantum yields (QY), determined by using quinine sulfate as a standard, are $29.5 \%, 33.9 \%, 9.3 \%$ and $10.2 \%$ for $G T$-C-dots, $C T$-C-dots, $G D$-C-dots and $C D$-C-dots, respectively, which shows that the QYs of C-dots prepared in the presence of TTDDA is higher than those of C-dots prepared in the presence of dopamine. These results indicate that TTDDA 
and dopamine not only play key roles in the emission wavelength of the prepared C-dots, but also contribute to the QY.

Contribution of the reaction precursors to PL properties of these C-dots were further investigated by using the samples obtained at different reaction times. Figure 4 shows the measured 2D-PL topographical maps of C-dots. Clearly, the excitation and emission properties of C-dots prepared in the presence of TTDDA are different from those prepared in the presence of dopamine. For GT-C-dots and CT-C-dots, the emission wavelengths do not change with reaction time in the investigated time frame. However, the emission wavelengths of $G D$-C-dots and $C D$-C-dots red shift with prolonging the reaction time, as shown in Figure 4C and D. These finding indicate that likely a longer time is needed for carbonization of the complexes formed with dopamine. Interestingly, the evolution of emission wavelength of $C D$-C-dots with reaction time is different from that of GD-C-dots, as shown in Figure 4D. After one hour of the reaction, the obtained $C D$-C-dots show two emission peaks located around $410 \mathrm{~nm}$ and $510 \mathrm{~nm}$, respectively. With the increase of reaction time, the emission peak at $410 \mathrm{~nm}$ gradually disappeared and the peak at $510 \mathrm{~nm}$ red shifts, which indicates the size of C-dots become more even and the chemical features are more uniform. Additionally, it can be seen from Figure 4 that the emission of all the of C-dots show little dependence on the excitation wavelength in the tested excitation wavelength range, which is rather different from that previously reported excitation-dependent emission property of C-dots.[25, 31] This phenomenon should be due to the narrow size distribution and uniform chemical features on the surfaces of the prepared C-dot. In fact, the independence of emission on the excitation wavelength has also been observed in the Cdots prepared by non-enzymatic browning reaction of amino acids with glucose,[4] pyrolysis of PAN@PMMA core-shell nanoparticles[47] and electrooxidation of graphite.[48] 

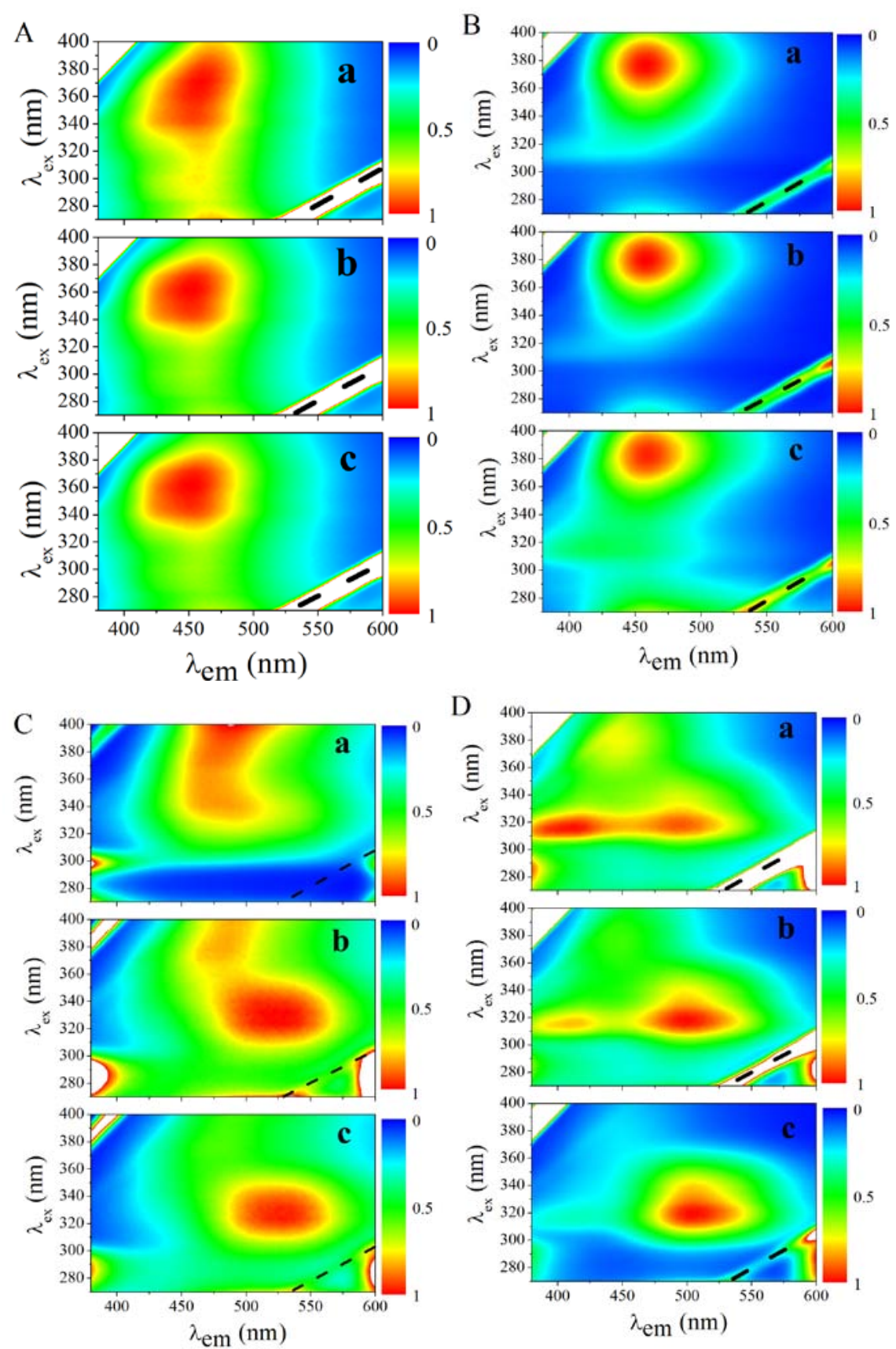

Figure 4 Fluorescence emission -excitation maps of (A) GT-C-dots, (B) CT-C-dots, (C) GD-Cdots and (D) $C D$-C-dots sampled at different reaction time: (a) $60 \mathrm{~min}$, (b) $100 \mathrm{~min}$, (c) $140 \mathrm{~min}$. The signals indicated by dashed lines are due to the second order diffraction of the excitation light. 
The compositions and structures of these four types of C-dots were characterized by FT-IR spectroscopy and elemental analysis. Figure 5 shows the obtained FT-IR spectra, which clearly identify the carboxyl group, both through the very broad $3300 \mathrm{~cm}^{-1} \mathrm{O}-\mathrm{H}$ stretching absorption and the $1697 \mathrm{~cm}^{-1} \mathrm{C}=\mathrm{O}$ stretching vibration for all four types of $\mathrm{C}$-dots. The absorption at 1610 $\mathrm{cm}^{-1}$ is assigned to amide derived from conversion of the carboxylic groups and the absorption at $1120 \mathrm{~cm}^{-1}$ was due to the $\mathrm{C}$-N vibration. The FT-IR spectra of $G D$-dots and $C D$-C-dots are more complex than those of $G T$-C-dots and $C T$-C-dots, especially in the fingerprint region, even the same carbon source used. That could be clearly attributed to the different nitrogen sources used. The elemental analysis results are given in Table 1. It is clear that the content of nitrogen in the $\mathrm{C}$-dots derived from TTDDA are much higher than those of C-dots derived from dopamine, which explains why the emission wavelengths of $G T$-C-dots and $C T$-C-dots are shorted than those of $G D$-C-dots and $C D$-C-dots. Because the relatively strong electron affinity of $\mathrm{N}$ atoms in the carbon nanomaterials typically causes the PL blue shift.[46, 49]

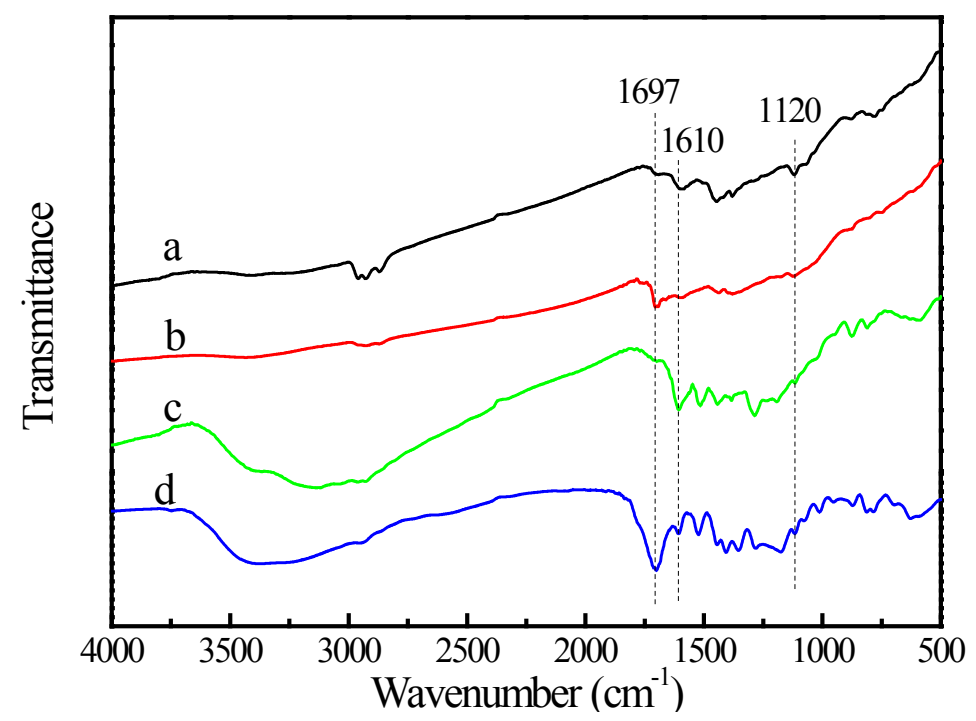

Figure 5 FT-IR spectra of (a) GT-C-dots, (b) $C T$-C-dots, (c) GD-C-dots and (d) $C D$-C-dots. 
Table 1 Elemental analysis results of the prepared C-dots.

\begin{tabular}{lcccc}
\hline & \multicolumn{4}{c}{ Composition (wt \%) } \\
\cline { 2 - 5 } & $\mathrm{N}$ & $\mathrm{C}$ & $\mathrm{H}$ & $\mathrm{O}$ \\
\hline$G T$-C-dots & 7.03 & 56.34 & 9.28 & 27.35 \\
$C T$-C-dots & 7.14 & 55.57 & 8.29 & 29.00 \\
$G D$-C-dots & 2.15 & 53.39 & 6.04 & 38.41 \\
$C D$-C-dots & 3.37 & 48.57 & 5.17 & 42.89 \\
\hline
\end{tabular}

Besides the effect of the reaction precursors on the optical properties of C-dots, further experiments were carried out to investigate the effect of reaction precursors on other physicochemical properties of the C-dots. We first studied the $\mathrm{pH}$-sensitivity of all four types of C-dots. The results are presented in Figure 6. All of the C-dots show certain sensitivity to variation in $\mathrm{pH}$, which is different from the commonly reported $\mathrm{pH}$-insensitive $\mathrm{C}$-dots.[50] When $\mathrm{pH}$ increased from 2 to 10, a decrease in PL intensities of GT-C-dots, $C T$-C-dots, GD-C-dots and $C D$-C-dots were $50 \%, 31 \%, 75 \%$ and $90 \%$, respectively. The pH sensitivity of C-dots is generally explained by the reversible protonation of amide and carboxylic groups on the surface of C-dots in acidic and alkali media which are relevant to the photoemission of C-dots.[51, 52] However, the observed higher $\mathrm{pH}$-sensitivities of $G D$-C-dots and $C D$-C-dots compared to $G T$-Cdots and $C T$-C-dots can not simply explained by protonation of amide and carboxylic acid groups. We suggest that the higher $\mathrm{pH}$-sensitivities could be due to existence of dopamine / dopamine analogues on the surface of $G D$-C-dots and $C D$-C-dots. At high $\mathrm{pH}$, the dopamine / dopamine analogues can be oxidized from hydroquinone structure to quinone structure by ambient $\mathrm{O}_{2}$. The quinone structure is a favorable electron acceptor and provides a non-radiative channel excited electrons, $[53,54]$ resulting in an increase in C-dots PL quenching. That would also explain the lower fluorescence quantum yields of $G D$-C-dots and $C D$-C-dots than that of 
GT-C-dots and CT-C-dots. In addition to the change in PL intensities, the emission wavelengths of these prepared C-dots are also blue shifted with the increase of $\mathrm{pH}$ value.
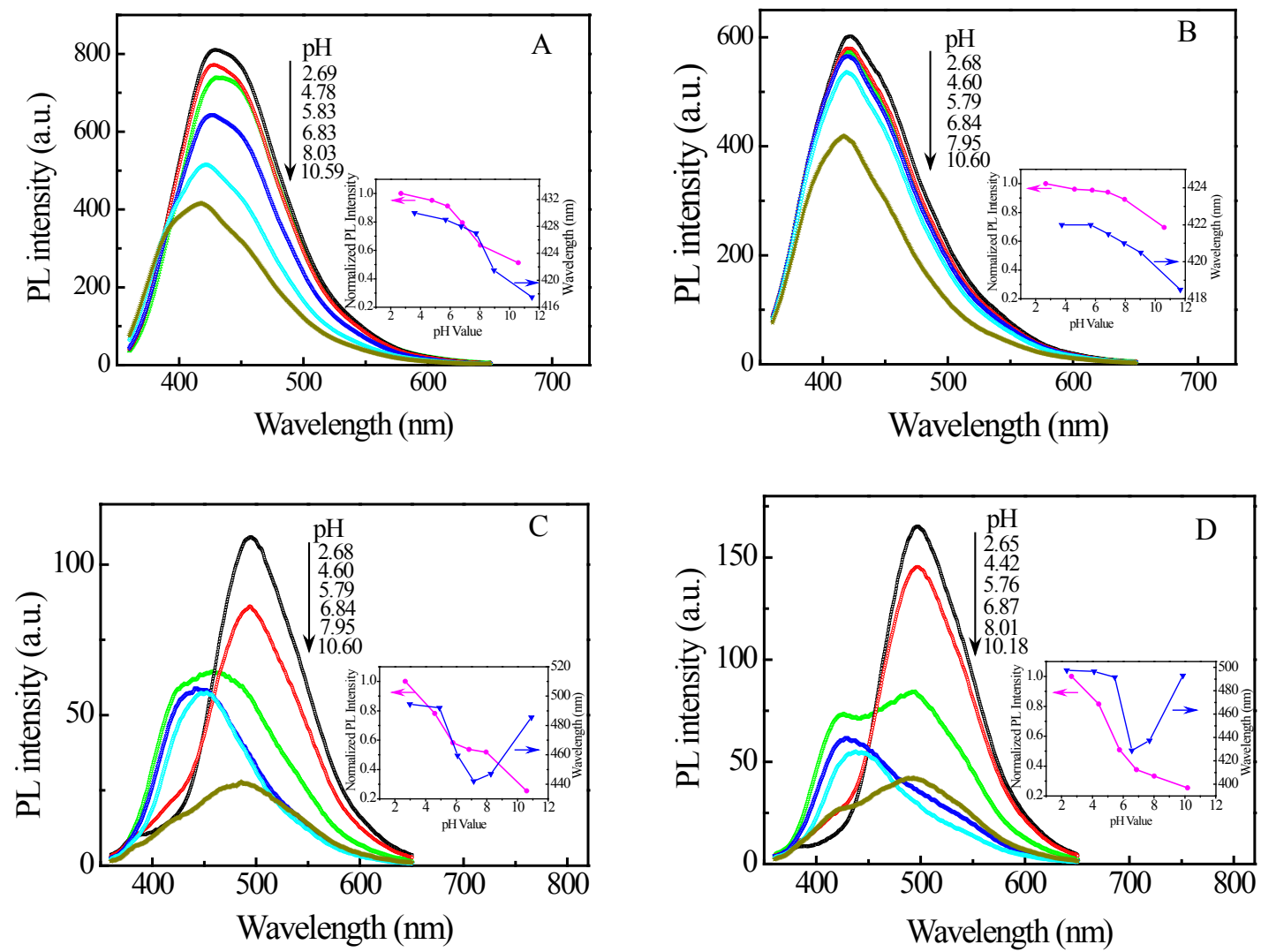

Figure 6 PL spectra of (A) GT-C-dots (B) $C T$-C-dots (C) $G D$-C-dots and (D) $C D$-C-dots at different $\mathrm{pH}$ values. Insets: Plot of the dependence of PL intensity and peak wavelength on $\mathrm{pH}$. The PL intensity is normalized to the lowest $\mathrm{pH}$ value.

Interestingly, $G D$-C-dots and $C D$-C-dots show electrochemical activities, as shown in Figure 7A and B. It is evident that oxidation and reduction peaks of $G D$-C-dots and $C D$-C-dots appeared during the potential scan. For $G D$-C-dots, the oxidation and reduction peaks at $\mathrm{pH} 7.4$ located at $0.34 \mathrm{~V}$ and $0.10 \mathrm{~V}$, respectively. For $C D$-C-dots, the oxidation and reduction peaks at $\mathrm{pH} 7.4$ located at $0.35 \mathrm{~V}$ and $0 \mathrm{~V}$, respectively. The electrochemical reversibility of $G D$-C-dots and $C D$-C-dots is worse compared to that of dopamine (shown in Figure S2 in Supporting 
Information). The peak potentials negatively shifted with an increase in $\mathrm{pH}$ of the solution, which is similar to the electrochemical behavior of dopamine.[55] The observed electrochemical activities of $G D$-C-dots and $C D$-C-dots further indicates the existence of dopamine / dopamine analogues on the surface of the C-dots. The shift of redox peak potentials would be expected, as the protonation states of two hydroxyl groups in dopamine change as a function of $\mathrm{pH} .[55]$ To the best of our knowledge, this is the first observation of luminescent C-dots with electrochemical activity. These results show that not only the optical properties but also other physical / chemical properties could be tuned by the reaction precursors, which paves a new way for the facile preparation of multi-functionalized C-dots.

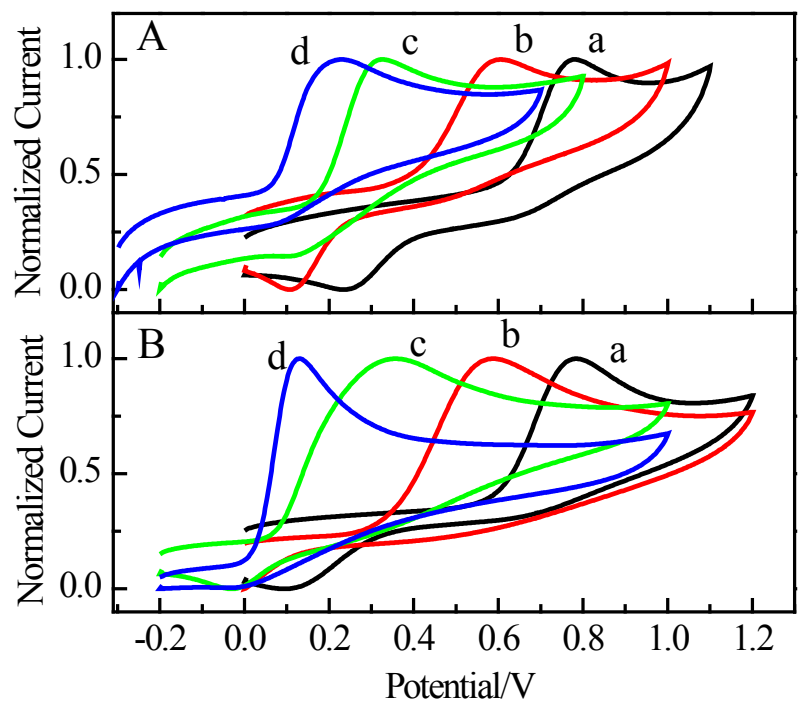

Figure 7 Normalized cyclic voltammograms of (A) $G D$-C-dots and (B) $C D$-C-dots obtained at (a) $\mathrm{pH} 1.4$, (b) $\mathrm{pH} 4.6$, (c) $\mathrm{pH} 7.4$ and $\mathrm{pH}$ 8.6. The scan rate is $50 \mathrm{mV} / \mathrm{s}$.

Inspired by the application of inorganic quantum dots as sensitizers in the solar cells, and by the advantages of broad absorption spectra, ease of synthesis and solution processability of Cdots, we investigated photovoltaic properties of the prepared C-dots as sensitizers. The fabrication and characterization of C-dots sensitized nanocrystalline $\mathrm{TiO}_{2}$ solar cells based on 
$\mathrm{I}_{3}{ }^{-} / \mathrm{I}^{-}$electrolyte are given in Supporting Information. The obtained photocurrent-voltage (I-V) plots of the fabricated solar cells under simulated AM 1.5 irradiation are shown in Figure 8. The photovoltaic characteristic parameters of short-circuit currents density $\left(\mathrm{I}_{\mathrm{sc}}\right)$, open-circuit potential $\left(\mathrm{V}_{\text {oc }}\right)$, fill factor $(\mathrm{FF})$, and photovoltaic conversion efficiency $(\eta)$ are listed in Table 2. As can be seen from Table 2, the values of $\mathrm{V}_{\mathrm{oc}}$ and $\mathrm{FF}$ are comparable to Ru-complex sensitized cells, however the current densities are much lower.[56] The low current density seems to be the main factor limiting the efficiencies of these cells, which is similar to the graphene dot sensitized nanocrystalline $\mathrm{TiO}_{2}$ solar cells.[57] The photovoltaic conversion efficiencies of solar cells based on the four types of $\mathrm{C}$-dots investigated are in the order of $G D$-C-dots $>C D$-C-dots $>C T$-C-dots $>$ GT-C-dots. The highest photovoltaic conversion efficiency of the solar cell based on GD-Cdots is $0.53 \%$, likely due to the wider absorption range, as shown in Figure 3. Although the efficiencies of these cells are quite low, our results illustrate that the photovoltaic properties of C-dots can also be tuned by choosing reaction precursors. The obtained photovoltaic conversion efficiency of $0.53 \%$ is nearly 5 times more than that of the reported nanocrystalline $\mathrm{TiO}_{2}$ solar cells based the C-dots prepared from the dehydration of a single molecule precursor $\gamma$ butyrolactone.[19] Work to improve the photovoltaic conversion efficiency is currently underway in our laboratory by using polysulfide based electrolytes to replace the $\mathrm{I}_{3}{ }^{-} / \mathrm{I}^{-}$ electrolyte and further functionalization of the C-dots with electron-donating or -withdrawing groups to improve the charge injection. 


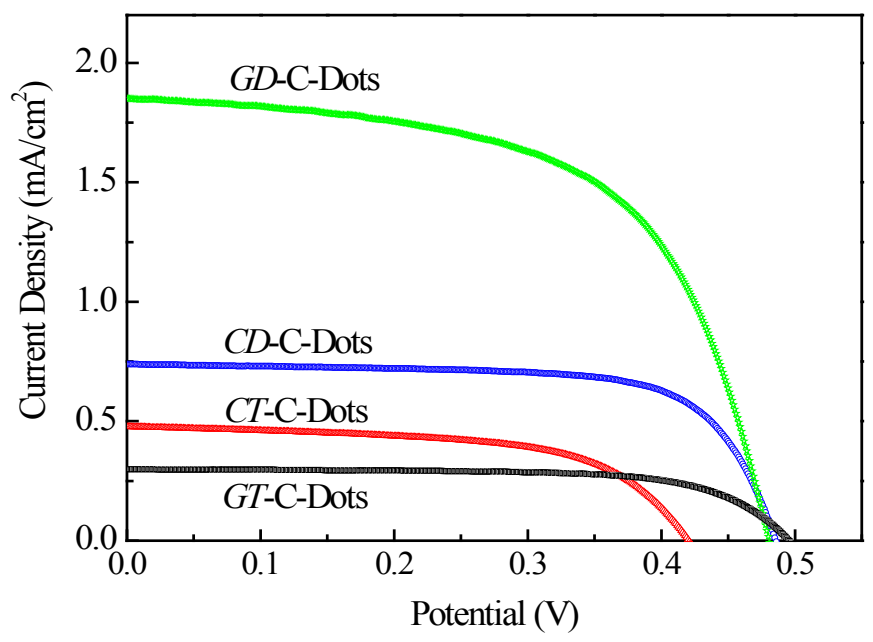

Figure 8 Current-voltage characteristics of nanocrystalline $\mathrm{TiO}_{2}$ solar cell sensitized by GT-Cdots, $C T$-C-dots, $G D$-C-dots and $C D$-C-dots

Table 2 Photovoltaic performance of the solar cells based on GT-C-dots, CT-C-dots, GD-C-dots and $C D$-C-dots under AM $1.5 \mathrm{G}$ illumination.

\begin{tabular}{lcccc}
\hline Solar Cells & $\begin{array}{c}\mathrm{I}_{\mathrm{sc}} \\
\left(\mathrm{mA} \bullet \mathrm{cm}^{-2}\right)\end{array}$ & $\begin{array}{c}\mathrm{V}_{\mathrm{oc}} \\
(\mathrm{V})\end{array}$ & $\begin{array}{c}\text { Fill factor (FF } \\
\%)\end{array}$ & $\begin{array}{c}\text { Efficiency } \\
(\eta \%)\end{array}$ \\
\hline$G T$-C-dots & 0.30 & 0.495 & 67.9 & 0.10 \\
CT-C-dots & 0.46 & 0.420 & 61.2 & 0.12 \\
$G D$-C-dots & 1.85 & 0.480 & 59.3 & 0.53 \\
CD-C-dots & 0.74 & 0.485 & 70.0 & 0.25 \\
\hline
\end{tabular}

\section{Conclusions}

In this work, four types of luminescent nitrogen-doped C-dots were prepared by using glucose or citric acid as carbon sources, and dopamine or TTDDA as nitrogen sources. The C-dots prepared in the presence of TTDDA possess much stronger PL (with QY up to $33.9 \%$ ) than those prepared in the presence of dopamine. However, the C-dots obtained with dopamine show better $\mathrm{pH}$ sensitivity due to the existence of dopamine / dopamine analogues that are electron 
acceptors. These results indicate that nitrogen source plays a key role in the properties of obtained C-dots. Furthermore, the C-dots obtained with dopamine exhibit interesting, $\mathrm{pH}$ dependent, electrochemical activities which indicate multiple functionalized C-dots can be prepared in a one-step by appropriately choosing reaction precursors. As a potential alternative to the dye-sensitized solar cells, the prepared C-dots were used as sensitizers to construct nanocrystalline $\mathrm{TiO}_{2}$ solar cell. The highest photovoltaic conversion efficiency was $0.53 \%$. These results clearly demonstrate that optical and other physicochemical properties of C-dots can be tuned by the use of appropriate reaction precursors.

\section{Acknowledgment}

This work was supported by the 973 Program No. 2014CB921104, the NSF of China (Grant Nos. 61201071, 61306020) and Program of Shanghai Subject Chief Scientist.

\section{Supplementary data}

Supplementary data associated with this article can be found in the online version, at

\section{References}

[1] Y. Fang, S. Guo, D. Li, C. Zhu, W. Ren, S. Dong, et al., Easy Synthesis and Imaging Applications of Cross-Linked Green Fluorescent Hollow Carbon Nanoparticles, ACS Nano 6 (2011) 400-409.

[2] Y. Yang, J. Cui, M. Zheng, C. Hu, S. Tan, Y. Xiao, et al., One-step synthesis of aminofunctionalized fluorescent carbon nanoparticles by hydrothermal carbonization of chitosan, Chem. Commun. 48 (2012) 380-382.

[3] H. Li, X. He, Y. Liu, H. Yu, Z. Kang, S.-T. Lee, Synthesis of fluorescent carbon nanoparticles directly from active carbon via a one-step ultrasonic treatment, Mater. Res. Bull. 
46 (2011) 147-151.

[4] W. Wei, C. Xu, L. Wu, J. Wang, J. Ren, X. Qu, Non-Enzymatic-Browning-Reaction: A Versatile Route for Production of Nitrogen-Doped Carbon Dots with Tunable Multicolor Luminescent Display, Sci. Rep. 4 (2014) 3564.

[5] Q.-L. Zhao, Z.-L. Zhang, B.-H. Huang, J. Peng, M. Zhang, D.-W. Pang, Facile preparation of low cytotoxicity fluorescent carbon nanocrystals by electrooxidation of graphite, Chem. Commun. 41 (2008) 5116-5118.

[6] J.-H. Liu, S.-T. Yang, X.-X. Chen, H. Wang, Fluorescent carbon dots and nanodiamonds for biological imaging: preparation, application, pharmacokinetics and toxicity, Curr. Drug Metab. 13 (2012) 1046-1056.

[7] Y. Wang, P. Anilkumar, L. Cao, J.-H. Liu, P.G. Luo, K.N. Tackett, II, et al., Carbon dots of different composition and surface functionalization: cytotoxicity issues relevant to fluorescence cell imaging, Exp. Biol. Med. 236 (2011) 1231-1238.

[8] X. Wang, L. Cao, S.T. Yang, F. Lu, M.J. Meziani, L. Tian, et al., Bandgap-like strong fluorescence in functionalized carbon nanoparticles, Angew. Chem. 49 (2010) 5310-5314.

[9] Y. Guo, Z. Wang, H. Shao, X. Jiang, Hydrothermal synthesis of highly fluorescent carbon nanoparticles from sodium citrate and their use for the detection of mercury ions, Carbon 52 (2013) 583-589.

[10] S.N. Baker, G.A. Baker, Luminescent Carbon Nanodots: emergent Nanolights, Angew. Chem., Int. Ed. 49 (2010) 6726-6744, S6726/6721-S6726/6725.

[11] Y. Li, C. Luo, C. Jiang, R. Huang, Y. Wang, H. Peng, et al., Luminescent carbon nanoparticles as a donor for the FRET-based detection of oligonucleotide hybridization, RSC Advances 4 (2014) 25201-25204. 
[12] W. Shi, X. Li, H. Ma, A Tunable Ratiometric pH Sensor Based on Carbon Nanodots for the Quantitative Measurement of the Intracellular pH of Whole Cells, Angew. Chem., Int. Ed. 51 (2012) 6432-6435.

[13] S.K. Bhunia, A. Saha, A.R. Maity, S.C. Ray, N.R. Jana, Carbon Nanoparticle-based Fluorescent Bioimaging Probes, Sci. Rep. 3 (2013) 1473.

[14] Y. Fang, S. Guo, D. Li, C. Zhu, W. Ren, S. Dong, et al., Easy Synthesis and Imaging Applications of Cross-Linked Green Fluorescent Hollow Carbon Nanoparticles, ACS Nano 6 (2012) 400-409.

[15] L. Cao, X. Wang, M.J. Meziani, F. Lu, H. Wang, P.G. Luo, et al., Carbon Dots for Multiphoton Bioimaging, J. Am. Chem. Soc. 129 (2007) 11318-11319.

[16] C. Liu, P. Zhang, X. Zhai, F. Tian, W. Li, J. Yang, et al., Nano-carrier for gene delivery and bioimaging based on carbon dots with PEI-passivation enhanced fluorescence, Biomaterials 33 (2012) 3604-3613.

[17] X. Michalet, F.F. Pinaud, L.A. Bentolila, J.M. Tsay, S. Doose, J.J. Li, et al., Quantum Dots for Live Cells, in Vivo Imaging, and Diagnostics, Science 307 (2005) 538-544.

[18] X. Guo, C.-F. Wang, Z.-Y. Yu, L. Chen, S. Chen, Facile access to versatile fluorescent carbon dots toward light-emitting diodes, Chem. Commun. 48 (2012) 2692-2694.

[19] P. Mirtchev, E.J. Henderson, N. Soheilnia, C.M. Yip, G.A. Ozin, Solution phase synthesis of carbon quantum dots as sensitizers for nanocrystalline TiO2 solar cells, J. Mater. Chem. 22 (2012) 1265-1269.

[20] Y.-P. Sun, B. Zhou, Y. Lin, W. Wang, K.A.S. Fernando, P. Pathak, et al., Quantum-Sized Carbon Dots for Bright and Colorful Photoluminescence, J. Am. Chem. Soc. 128 (2006) 77567757. 
[21] H. Liu, T. Ye, C. Mao, Fluorescent carbon nanoparticles derived from candle soot, Angew Chem Int Ed Engl 46 (2007) 6473-6475.

[22] H. Peng, J. Travas-Sejdic, Simple Aqueous Solution Route to Luminescent Carbogenic Dots from Carbohydrates, Chem. Mater. 21 (2009) 5563-5565.

[23] Z.-C. Yang, X. Li, J. Wang, Intrinsically fluorescent nitrogen-containing carbon nanoparticles synthesized by a hydrothermal process, Carbon 49 (2011) 5207-5212.

[24] J.R. Neabo, C. Vigier-Carriere, S. Rondeau-Gagne, J.-F. Morin, Room-temperature synthesis of soluble, fluorescent carbon nanoparticles from organogel precursors, Chem. Commun. 48 (2012) 10144-10146.

[25] L. Bao, Z.-L. Zhang, Z.-Q. Tian, L. Zhang, C. Liu, Y. Lin, et al., Electrochemical Tuning of Luminescent Carbon Nanodots: From Preparation to Luminescence Mechanism, Adv. Mater. 23 (2011) 5801-5806.

[26] H. Li, X. He, Z. Kang, H. Huang, Y. Liu, J. Liu, et al., Water-Soluble Fluorescent Carbon Quantum Dots and Photocatalyst Design, Angew. Chem. Int. Ed. 49 (2010) 4430-4434.

[27] H. Peng, J. Travas-Sejdic, Simple Aqueous Solution Route to Luminescent Carbogenic Dots from Carbohydrates, Chem. Mater. 21 (2009) 5563-5565.

[28] J. Jiang, Y. He, S. Li, H. Cui, Amino acids as the source for producing carbon nanodots: microwave assisted one-step synthesis, intrinsic photoluminescence property and intense chemiluminescence enhancement, Chem. Commun. 48 (2012) 9634-9636.

[29] Z. Zhang, J. Hao, J. Zhang, B. Zhang, J. Tang, Protein as the source for synthesizing fluorescent carbon dots by a one-pot hydrothermal route, RSC Adv. 2 (2012) 8599-8601.

[30] J. Wang, C.-F. Wang, S. Chen, Amphiphilic Egg-Derived Carbon Dots: Rapid Plasma Fabrication, Pyrolysis Process, and Multicolor Printing Patterns, Angew. Chem., Int. Ed. 51 
(2012) 9297-9301.

[31] S. Liu, J. Tian, L. Wang, Y. Zhang, X. Qin, Y. Luo, et al., Hydrothermal Treatment of

Grass: A Low-Cost, Green Route to Nitrogen-Doped, Carbon-Rich, Photoluminescent Polymer Nanodots as an Effective Fluorescent Sensing Platform for Label-Free Detection of $\mathrm{Cu}$ (II) Ions, Adv. Mater. 24 (2012) 2037-2041.

[32] M.J. Krysmann, A. Kelarakis, E.P. Giannelis, Photoluminescent carbogenic nanoparticles directly derived from crude biomass, Green Chem. 14 (2012) 3141-3145.

[33] H. Li, Z. Kang, Y. Liu, S.-T. Lee, Carbon nanodots: synthesis, properties and applications, J. Mater. Chem. 22 (2012) 24230-24253.

[34] X. Li, S. Zhang, S.A. Kulinich, Y. Liu, H. Zeng, Engineering surface states of carbon dots to achieve controllable luminescence for solid-luminescent composites and sensitive $\mathrm{Be} 2+$ detection, Sci. Rep. 4 (2014) 4976.

[35] Z.-C. Yang, M. Wang, A.M. Yong, S.Y. Wong, X.-H. Zhang, H. Tan, et al., Intrinsically fluorescent carbon dots with tunable emission derived from hydrothermal treatment of glucose in the presence of monopotassium phosphate, Chem. Commun. 47 (2011) 11615-11617.

[36] J. Zhou, X. Zhou, R. Li, X. Sun, Z. Ding, J. Cutler, et al., Electronic structure and luminescence center of blue luminescent carbon nanocrystals, Chem. Phys. Lett. 474 (2009) 320324.

[37] A.B. Bourlinos, A. Stassinopoulos, D. Anglos, R. Zboril, M. Karakassides, E.P. Giannelis, Surface Functionalized Carbogenic Quantum Dots, Small 4 (2008) 455-458.

[38] J. Zhou, C. Booker, R. Li, X. Zhou, T.-K. Sham, X. Sun, et al., An Electrochemical Avenue to Blue Luminescent Nanocrystals from Multiwalled Carbon Nanotubes (MWCNTs), J. Am. Chem. Soc. 129 (2007) 744-745. 
[39] M.J. Krysmann, A. Kelarakis, P. Dallas, E.P. Giannelis, Formation Mechanism of Carbogenic Nanoparticles with Dual Photoluminescence Emission, J. Am. Chem. Soc. 134 (2011) $747-750$.

[40] R. Shen, K. Song, H. Liu, Y. Li, H. Liu, Fluorescence Enhancement and Radiolysis of Carbon Dots through Aqueous $\gamma$ Radiation Chemistry, J. Phys. Chem. C 116 (2012) 15826-15832. [41] Z. Ma, H. Ming, H. Huang, Y. Liu, Z. Kang, One-step ultrasonic synthesis of fluorescent N-doped carbon dots from glucose and their visible-light sensitive photocatalytic ability, New J. Chem. 36 (2012) 861-864.

[42] X. Jia, J. Li, E. Wang, One-pot green synthesis of optically pH-sensitive carbon dots with upconversion luminescence, Nanoscale 4 (2012) 5572-5575.

[43] L. Tang, R. Ji, X. Cao, J. Lin, H. Jiang, X. Li, et al., Deep Ultraviolet Photoluminescence of Water-Soluble Self-Passivated Graphene Quantum Dots, ACS Nano 6 (2012) 5102-5110.

[44] K. Habiba, V.I. Makarov, J. Avalos, M.J.F. Guinel, B.R. Weiner, G. Morell, Luminescent graphene quantum dots fabricated by pulsed laser synthesis, Carbon 64 (2013) 341-350.

[45] L. Li, G. Wu, G. Yang, J. Peng, J. Zhao, J.-J. Zhu, Focusing on luminescent graphene quantum dots: current status and future perspectives, Nanoscale 5 (2013) 4015-4039.

[46] Y. Li, Y. Zhao, H. Cheng, Y. Hu, G. Shi, L. Dai, et al., Nitrogen-Doped Graphene Quantum Dots with Oxygen-Rich Functional Groups, J. Am. Chem. Soc. 134 (2012) 15-18.

[47] Y. Wang, L. Dong, R. Xiong, A. Hu, Practical access to bandgap-like N-doped carbon dots with dual emission unzipped from PAN@PMMA core-shell nanoparticles, J. Mater. Chem. C 1 (2013) 7731-7735.

[48] Q.-L. Zhao, Z.-L. Zhang, B.-H. Huang, J. Peng, M. Zhang, D.-W. Pang, Facile preparation of low cytotoxicity fluorescent carbon nanocrystals by electrooxidation of graphite, 
Chem. Commun. (2008) 5116-5118.

[49] K. Gong, F. Du, Z. Xia, M. Durstock, L. Dai, Nitrogen-Doped Carbon Nanotube Arrays with High Electrocatalytic Activity for Oxygen Reduction, Science 323 (2009) 760-764.

[50] O.S. Wolfbeis, An overview of nanoparticles commonly used in fluorescent bioimaging, Chem. Soc. Rev. 44 (2015) 4743-4768.

[51] L. Shen, L. Zhang, M. Chen, X. Chen, J. Wang, The production of $p H-s e n s i t i v e$ photoluminescent carbon nanoparticles by the carbonization of polyethylenimine and their use for bioimaging, Carbon 55 (2013) 343-349.

[52] D. Pan, J. Zhang, Z. Li, C. Wu, X. Yan, M. Wu, Observation of pH-, solvent-, spin-, and excitation-dependent blue photoluminescence from carbon nanoparticles, Chem. Commun. 46 (2010) 3681-3683.

[53] X. Ji, N.S. Makarov, W. Wang, G. Palui, I. Robel, H. Mattoussi, Tuning the Redox Coupling between Quantum Dots and Dopamine in Hybrid Nanoscale Assemblies, J. Phys. Chem. C 119 (2015) 3388-3399.

[54] I.L. Medintz, M.H. Stewart, S.A. Trammell, K. Susumu, J.B. Delehanty, B.C. Mei, et al., Quantum-dot/dopamine bioconjugates function as redox coupled assemblies for in vitro and intracellular pH sensing, Nat. Mater. 9 (2010) 676-684.

[55] E. Laviron, Electrochemical reactions with protonations at equilibrium. Part X. The kinetics of the p-benzoquinone/hydroquinone couple on a platinum electrode, J. Electroanal. Chem. Interfacial Electrochem. 164 (1984) 213-227.

[56] M.K. Nazeeruddin, A. Kay, I. Rodicio, R. Humphry-Baker, E. Mueller, P. Liska, et al., Conversion of light to electricity by cis-X2bis(2,2'-bipyridyl-4,4'-dicarboxylate)ruthenium(II) charge-transfer sensitizers ( $\mathrm{X}=\mathrm{Cl}-, \mathrm{Br}-, \mathrm{I}-, \mathrm{CN}-$, and $\mathrm{SCN}-)$ on nanocrystalline titanium dioxide 
electrodes, J. Am. Chem. Soc. 115 (1993) 6382-6390.

[57] X. Yan, X. Cui, B. Li, L.-s. Li, Large, Solution-Processable Graphene Quantum Dots as Light Absorbers for Photovoltaics, Nano Lett. 10 (2010) 1869-1873. 